\title{
Proinsulin peptide promotes autoimmune diabetes in a novel HLA-DR3-DQ2-transgenic murine model of spontaneous disease
}

\author{
Johan Verhagen $^{1}$ (D) Norkhairin Yusuf ${ }^{1}$ • Emma L. Smith ${ }^{2} \cdot$ Emily M. Whettlock $^{1,3} \cdot$ Kerina Naran $^{1} \cdot$ Sefina Arif $^{1}$. \\ Mark Peakman ${ }^{1,4}$
}

Received: 5 June 2019 / Accepted: 7 August 2019/Published online: 14 October 2019

(C) The Author(s) 2019

\begin{abstract}
Aims/hypothesis The molecular basis for the pathological impact of specific HLA molecules on autoimmune diseases such as type 1 diabetes remains unclear. Recent natural history studies in children have indicated a link between specific HLA genotypes and the first antigenic target against which immune responses develop. We set out to examine this link in vivo by exploring the diabetogenicity of islet antigens on the background of a common diabetes-associated HLA haplotype.

Methods We generated a novel HLA-transgenic mouse model that expresses high-risk genes for type 1 diabetes (DRB1*03:01$D Q A 1 * 05: 01-D Q B 1 * 02: 01)$ as well as human $C D 80$ under the rat insulin promoter and human $C D 4$, on a $\mathrm{C} 57 \mathrm{BL} / 6$ background. Adjuvanted antigen priming was used to reveal the diabetogenicity of candidate antigens and peptides.

Results HLA-DR3-DQ2 ${ }^{+}$huCD4 ${ }^{+}$IA/IE ${ }^{-/}$RIP.B $7.1^{+}$mice spontaneously developed autoimmune diabetes (incidence $46 \%$ by 35 weeks of age), accompanied by numerous hallmarks of human type 1 diabetes (autoantibodies against GAD65 and proinsulin; pancreatic islet infiltration by $\mathrm{CD}^{+}, \mathrm{CD}^{+} \mathrm{B} 220^{+}, \mathrm{CD} 11 \mathrm{~b}^{+}$and $\mathrm{CD} 11 \mathrm{c}^{+}$immune cells). Disease was markedly accelerated and had deeper penetrance after adjuvanted antigen priming with proinsulin (mean onset 11 weeks and incidence $100 \%$ by 20 weeks post challenge). Moreover, the diabetogenic effect of proinsulin located to the 15-residue B29-C11 region.

Conclusions/interpretation Our study identifies a proinsulin-derived peptide region that is highly diabetogenic on the HLADR3-DQ2 background using an in vivo model. This approach and the peptide region identified may have wider implications for future studies of human type 1 diabetes.
\end{abstract}

Keywords HLA-DQ2 · HLA-DR3 · Mouse model · Proinsulin · Type 1 diabetes

\section{Abbreviations}

IA-2 Islet antigen-2

RIP Rat insulin promoter

Electronic supplementary material The online version of this article (https://doi.org/10.1007/s00125-019-04994-8) contains peer-reviewed but unedited supplementary material, which is available to authorised users.

Mark Peakman

mark.peakman@kcl.ac.uk

1 School of Immunology and Microbial Sciences, Faculty of Life Sciences \& Medicine, King's College London, 2nd Floor Borough Wing, Guy's Hospital, Great Maze Pond, London SE1 9RT, UK

2 UCB Pharma Ltd, Slough, UK

3 Present address: Department of Metabolism, Digestion and Reproduction, Chelsea \& Westminster Hospital, London, UK

4 Institute of Diabetes, Endocrinology and Obesity, King's Health Partners, London, UK
TEDDY The Environmental Determinants of Diabetes in the Young

\section{Introduction}

Type 1 diabetes, like all autoimmune diseases, results from the inappropriate activation of the immune system in response to autoantigen encounter. Although the condition can be managed increasingly well clinically, it still impacts negatively on quality of life and life expectancy $[1,2]$. The reasons for the loss of selftolerance in type 1 diabetes remain debated but it is well established that the HLA system constitutes a major genetic risk factor. HLA-DR3-DQ2 (DRB1*03:01-DQA1*05:01$\mathrm{DQB} 1 * 02: 01)$ is the most common haplotype found in individuals with type 1 diabetes, at a frequency of around 34\% [3], and 


\section{Research in context}

\section{What is already known about this subject?}

- HLA-DR3-DQ2 is the most common human haplotype associated with type 1 diabetes

- HLA haplotype determines the specificity of autoimmune responses

What is the key question?

- What is/are the dominant islet-derived antigen(s) that drive a diabetogenic immune response on a specific HLA background and which are the required, dominant peptide regions?

What are the new findings?

- HLA-DR3-DQ2+huCD4+IA/IE-/-RIP.B7.1 ${ }^{+}$mice develop spontaneous autoimmune diabetes, which shares many characteristics with human type 1 diabetes

- Adjuvanted priming with proinsulin accelerates the onset and aggravates the incidence of diabetes in this model

- The diabetogenic effect of proinsulin can be pinpointed to a previously unreported region covered by a 15 -mer peptide sequence that commences at the $\mathrm{C}$-terminal end of the B-chain

How might this impact on clinical practice in the foreseeable future?

- The identification of disease-driving epitopes is essential for understanding the aetiology of diabetes and thus may affect the clinical approach to managing the autoimmune dysfunction that underlies human type 1 diabetes

thus delineates a major, identifiable disease cohort. Given the antigen-presenting properties of HLA molecules, it is assumed that the diabetes risk associated with HLA-DR3-DQ2 relates to the selective presentation of peptide epitopes of potentially diabetogenic autoantigens. Therefore, identification of these autoantigens and specific disease-determining regions is an important step towards understanding disease aetiology.

Attempts to discover disease-relevant epitopes in clinical studies generally involve the elution or prediction of peptides bound to candidate HLA molecules on antigen-presenting cells and/or in vitro examination of T cell responses in individuals with type 1 diabetes. This approach has generated several potentially relevant HLA-DR3-DQ2-restricted epitopes from a number of islet antigens, including GAD65 and islet antigen-2 (IA-2) [4]. Interestingly, proinsulin, generally considered to be involved in the early stages of type 1 diabetes in both humans and mice [5, 6], has not been identified as a robust source of diabetes-related epitopes presented by HLA-DR3-DQ2. One recent study, however, has highlighted regions of proinsulin C-peptide as generating HLA-DQ2-restricted $\mathrm{CD}^{+} \mathrm{T}$ cell responses in individuals with type 1 diabetes [7]. As an alternative approach to understanding autoantigen-HLA interactions, The Environmental Determinants of Diabetes in the Young (TEDDY) study has investigated the natural history of the emergence of autoantibodies on specific HLA backgrounds, revealing that in HLADR3-DQ2 individuals GAD65 tends to be the first of the studied islet antigens against which autoantibodies are formed, while in HLA-DR4-DQ8 individuals it is insulin [8, 9]. These studies potentially provide important clues on early events related to loss of immunological tolerance during the development of type 1 diabetes but are less incisive regarding questions of antigen and epitope diabetogenicity.

To begin to address this issue more directly, we have opted for an in vivo approach to epitope discovery, using diabetes-susceptible, HLA-transgenic mice. We examined disease relevance of antigens and peptides by their ability to promote the development of autoimmune diabetes in susceptible animals. The HLA-DR3DQ2-transgenic mouse that we used as our base does not develop diabetes spontaneously [10], so we sought a genetic modification that would augment disease susceptibility. Moreover, through the application of adjuvanted antigen priming as a mechanism to accelerate and extend diabetes penetrance, we sought to demonstrate the importance of specific islet antigens as a diabetogenic driver on the HLA-DR3-DQ2 background.

\section{Methods}

Animals HLA-DR3-DQ2-transgenic mice (C57BL/6- $H 2^{d l A b 1-}$ ${ }^{E a} \mathrm{Tg}(\mathrm{Cd} 4-\mathrm{CD} 4) 2362 \mathrm{Litt} \mathrm{Tg}$ (HLA DR3-DQ2)303, described previously [10], and obtained from J. McCluskey [University of Melbourne, VIC, Australia]) were crossed with RIP-B7.1transgenic mice (B6.Cg-Tg(Ins2-CD80)3B7Flv/Orl; ID 00216; EMMA, Orleans, France) in order to obtain HLA-DR3$\mathrm{DQ} 2^{+}$huCD4 ${ }^{+} \mathrm{IA} / \mathrm{IE}^{-/}$RIP.B7. $1^{+}$mice, hereafter referred to as DR3DQ2×RIP-B7.1. DR4×RIP-B7.1 mice, generated by crossing B6.129S2-H2-Ab1 $1^{\text {tm } 1 \text { Gru }} \mathrm{Tg}$ (HLA-DRA/H2-Ea,HLADRB1*0401/H2-Eb)1Kito mice (Taconic, Germantown, MD, 
USA) with B6.Cg-Tg(Ins2-CD80)3B7Flv/Orl mice, were described previously [11]. All mice were kept under specificpathogen-free conditions, in individually ventilated cages, at the King's College London Biological Services Unit on $12 \mathrm{~h}$ lightdark cycles with food and water provided ad libitum. Experiments were conducted in accordance with UK Home Office regulations under a project licence held by M. Peakman. All work was subject to assessment and approved locally by Guy's Animal Welfare and Ethical Review Board (AWERB).

Priming antigens All murine proinsulin-2 peptides were custom manufactured by either Almac (Edinburgh, UK) or GLS Biochem (Shanghai, China) at $>95 \%$ purity. The 377 -aminoacid C-terminal fragment of human IA-2 was produced by ProteoGenix (Schiltigheim, France). Recombinant human GAD65 (T cell GAD) was purchased from Diamyd Medical (Stockholm, Sweden). Predicted HLA binding cores and affinities were generated with the online IEDB analysis resource (http://tools.immuneepitope.org/mhcii/), using the NetMHCIIpan prediction method. Values given represent results as on 3 June 2019.

Induction and monitoring of diabetes All mice were monitored regularly for unprovoked glycosuria using Diastix strips (Bayer, Basel, Switzerland). Some mice were monitored weekly for hyperglycaemia, from the age of 6-7 weeks, by a minimal puncture of the tail vein at alternate sides of the tail and analysis using a OneTouch Verio meter and strips (Lifescan, High Wycombe, UK), in order to closely monitor progression towards spontaneous diabetes. Mice were considered diabetic following a blood glucose reading $>16.7 \mathrm{mmol} / 1$ $(300 \mathrm{mg} / \mathrm{dl})$ in addition to confirmed glycosuria. For disease acceleration experiments, mice were distributed over equal or similar sized groups as indicated to achieve a comparable spread of age and sex for each group. Experiments were not randomised or blinded. Mice (aged 6-14 weeks) were primed with $100 \mu \mathrm{g}$ of peptides or protein in TiterMax Gold adjuvant (TiterMax, Norcross, GA, USA) s.c. at the base of the tail and received a second dose s.c. distributed over the inguinal region on day 14. Mice received $200 \mathrm{ng}$ pertussis toxin (Sigma, Poole, UK) in PBS intraperitoneally (i.p.) on days 0 and 1 or 2. Mice were then monitored weekly for hyperglycaemia and for glycosuria. Immediately upon detection of diabetes, mice were euthanised humanely, according to ethical approval. No animals were excluded from the data unless they had to be culled before the end of the experiment for non-diabetesrelated welfare issues (e.g. overgrooming or fighting wounds), which were rare.

Histology Pancreases embedded in OCT compound (Cellpath, Newtown, UK) were frozen in isopentane (Sigma) cooled with liquid nitrogen. $10 \mu \mathrm{m}$ sections were fixed in acetone before first staining with rabbit anti-mouse insulin (Ab63820 [Abcam, Cambridge, UK], $2.5 \mu \mathrm{g} / \mathrm{ml}$ ) in PBS $0.5 \%$ BSA, detected with Vector ImmPress anti-rabbit AP (Vector Labs, Peterborough, UK), and developed with Vector ImmPress Red. Immune cells in the tissues were stained with biotinylated antibodies (all from Biolegend, San Diego, CA, USA) to murine CD4 (clone GK1.5, which also recognises human CD4, $2.5 \mu \mathrm{g} / \mathrm{ml}$ ), CD8 (clone 536.7, $2.5 \mu \mathrm{g} / \mathrm{ml}$ ), CD11b (clone M1/70, $0.25 \mu \mathrm{g} / \mathrm{ml}$ ), CD11c (clone N418, $0.625 \mu \mathrm{g} / \mathrm{ml}$ ), Ly6G (clone 1A8, $0.5 \mu \mathrm{g} / \mathrm{ml}$ ) and B220 (clone RA3-6B2, $0.625 \mu \mathrm{g} / \mathrm{ml}$ ) in PBS $0.5 \%$ BSA for $2 \mathrm{~h}$. The optimal concentration for each antibody was determined by serial dilution. Staining was detected using VectaStain R.T.U. Elite ABC reagent and DAB peroxidase substrate kit from Vector Labs. Nuclei were stained with Mayer's haematoxylin (Sigma), before mounting slides with VectaMount (Vector Labs). Images were acquired on a Zeiss Axiovert A1 microscope using the Zen (blue edition) software supplied (Zeiss, Cambridge, UK). No cropping or alteration of the images was made. See electronic supplementary material (ESM) Methods for insulitis scoring.

Autoantibody ELISA Proinsulin-2 peptides or recombinant human GAD65 were coated onto Maxisorp plates (Nunc, Roskilde, Denmark) in ELISA coating buffer (eBioscience/ ThermoFisher, Altrincham, UK). Diluted sera (in PBS containing 5\% BSA, Sigma) or controls (mouse IgG2a antiinsulin clone ICBTACLS and isotype [ThermoFisher], mouse IgG1 anti-GAD65 clone N-GAD65 and isotype [Biolegend]) were incubated for $2 \mathrm{~h}$ at room temperature prior to detection with biotin-anti-mouse IgG (clone Poly4053, $0.25 \mu \mathrm{g} / \mathrm{ml}$ in PBS 5\% BSA), streptavidin-horseradish peroxidase (1:2000 in PBS 5\% BSA) and high-sensitivity TMB solution (all from Biolegend) and read at $450 \mathrm{~nm}$. Titrated concentrations (1$1000 \mathrm{ng} / \mathrm{ml}$ ) of the relevant control antibody on each plate were used to normalise data and assign an arbitrary unit to serum antibody levels.

Statistical analysis All analyses were performed with GraphPad Prism 8 software (San Diego, CA, USA). For survival graphs, the Mantel-Cox logrank test was used to assess differences over the full course of the experiments. In other experiments, student's $t$ test or Dunnett's multiple comparison test were used for comparing two groups or more, respectively. A value of $p<0.05$ was considered statistically significant.

\section{Results}

Spontaneous-onset diabetes in DR3DQ2 $\times$ RIP-B7.1 mice Unlike the DR4×RIP-B7.1 model we described previously [11], which does not show spontaneous insulitis or diabetes, DR3DQ2×RIP-B7.1 mice develop diabetes spontaneously 
Fig. 1 DR3DQ2 $\times$ RIP-B7.1 mice develop immune-mediated diabetes spontaneously. (a) Mice were monitored weekly for diabetes from the age of 6

7 weeks until the age of 35 weeks (32 male, 24 female mice).

Difference not significant, male vs female mice (Mantel-Cox logrank test). (b) Representative images of immunohistochemical double staining of frozen pancreas sections from a mouse with confirmed diabetes. Insulin stained in red, immune cells in brown. Magnification $\times 20$. Arrows indicate $\mathrm{Ly}_{6 \mathrm{G}}{ }^{+}$cells. (cf) $\operatorname{IgG}$ autoantibody ELISAs for anti-human GAD65 (antihuGAD65) protein (c, d) and antimouse proinsulin-2 (anti-muPI2) overlapping 30-mer peptides (e, f). Sera obtained cross-sectionally from mice aged 8-23 weeks were analysed (c, e). Red horizontal lines indicate median. $n=10-22$ samples per age bracket. ELISAs were performed on the same samples, in parallel. $* p<0.05$ and $* * * p<0.001$ (Dunnett's multiple comparisons test). Autoantibody levels in DR3DQ2 $\times$ RIP-B7.1

(HLA-DR3DQ2) mice with confirmed diabetes ( $n=7$, aged 16-35 weeks at diagnosis) were compared with those in nondiabetic DR4×RIP-B7.1 (HLA-

DR4) mice ( $n=5$, aged 30 weeks) (d, f); not significant (Student's $t$ test). AU, arbitrary units

a

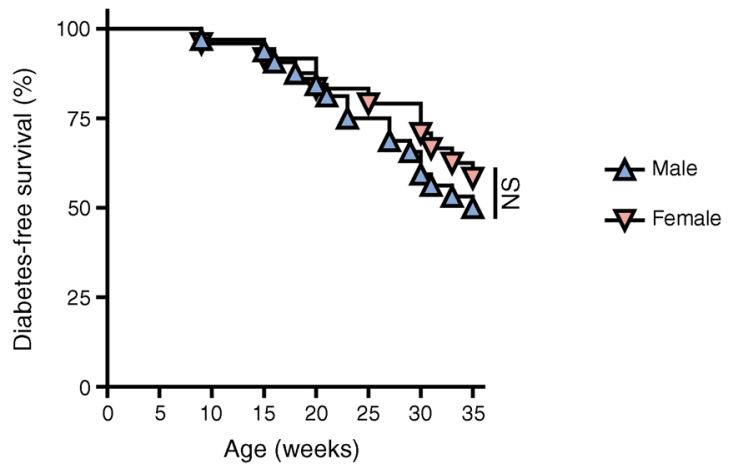

b
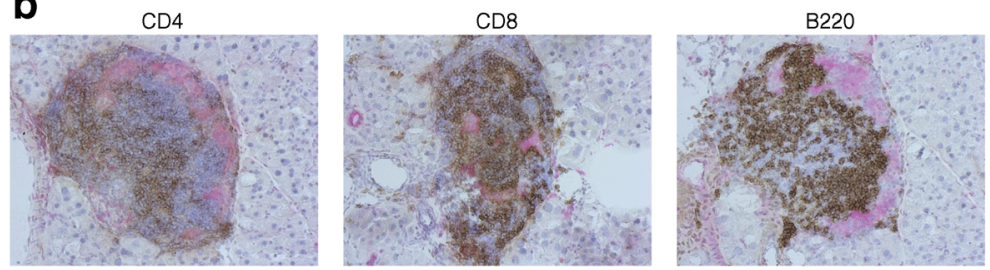

CD11b

CD11C
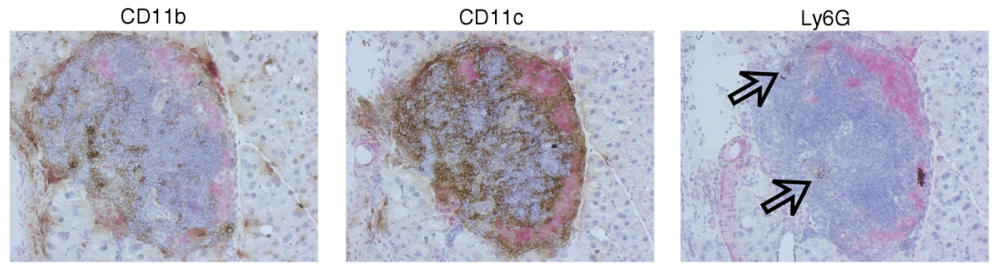

C

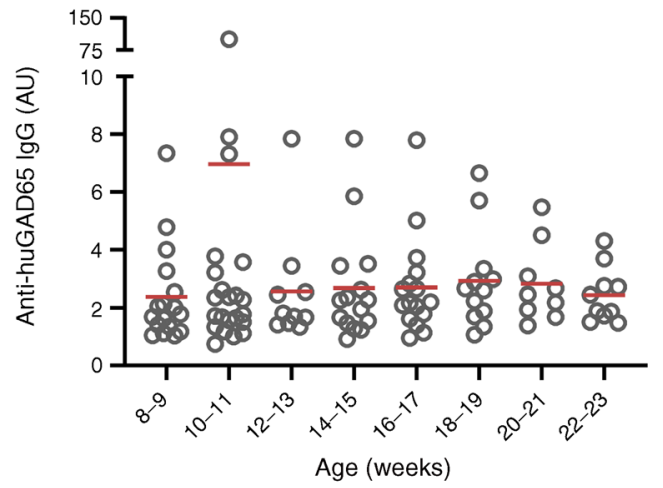

e

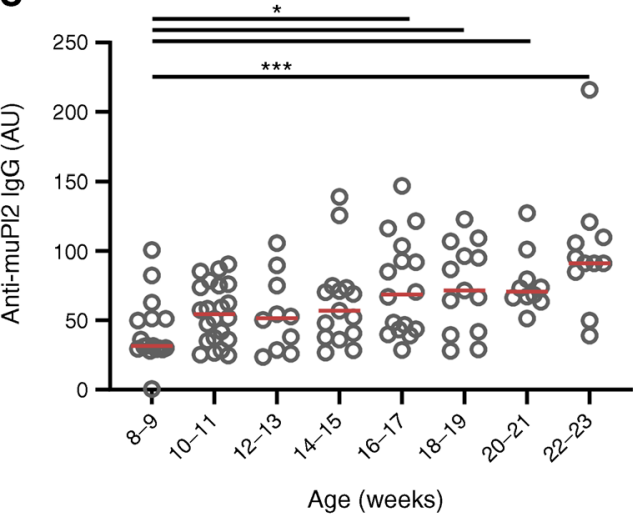

d

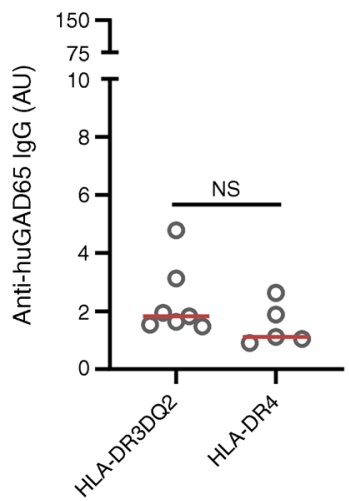

f

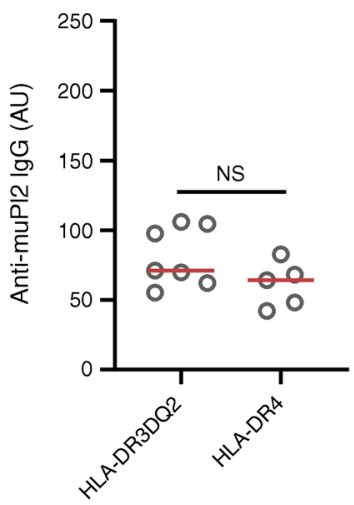


Fig. 2 Adjuvanted priming with murine proinsulin-2 peptides promotes diabetes. (a) DR3DQ2 $\times$ RIP-B7.1 mice aged 7-12 weeks were primed with $100 \mu \mathrm{g}$ of murine proinsulin-2 (muPI2) peptides (6 male, 4 female mice), human GAD65 (huGAD65) protein (5 male, 3 female mice) or the 377-aminoacid C-terminal region of IA-2 (huIA-2 fragment) (7 male, 2 female mice), and a PBS only control (5 male, 3 female mice) in TiterMax Gold adjuvant s.c. on days 0 and 14, with $200 \mathrm{ng}$ pertussis toxin administered i.p. on days 0 and 1 or 2 . Mice were monitored weekly for diabetes up to 20 weeks post prime. The grey dashed line indicates the expected level of spontaneous diabetes in mice aged 12 weeks on day 0 of the experiments (based on the data in Fig. 1 and normalised for sex). $* * p<0.01$ for muPI2 peptides vs huGAD65, and $* * * p$ $<0.001$ for muPI2 peptides vs both PBS and huIA-2 fragments (Mantel-Cox logrank test). (b-e) Blood glucose levels for each individual mouse in the group immunised with PBS (b), murine proinsulin-2 peptides (c), human GAD65 (d) or fragment of human IA-2 (e); key in (b) also applies to (c-e)
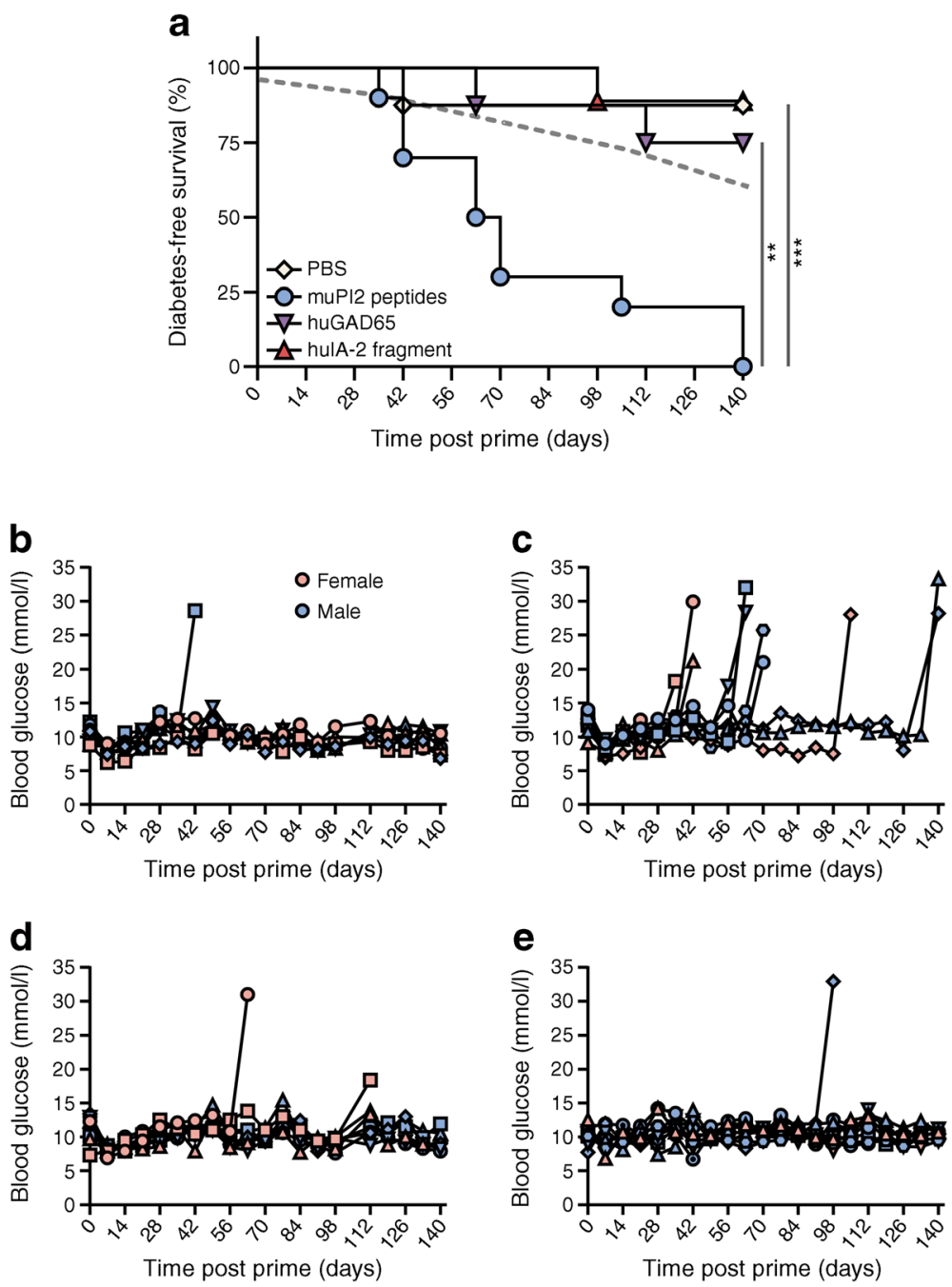

(Fig. 1a). Although both models demonstrate relatively high baseline levels of blood glucose at any age, only DR3DQ2 $\times$ RIP-B7.1 mice develop levels $>16.7 \mathrm{mmol} / \mathrm{l}$ in addition to glycosuria. By the age of 35 weeks, $46 \%$ of all mice monitored (26 out of 56) had developed autoimmune diabetes. In male and female mice, disease incidence (16/32 vs 10/24, respectively) and mean \pm SD age of onset were similar (24.2 \pm 7.3 weeks vs $24.8 \pm 8.5$, respectively). No obvious signs of other immune-mediated conditions, as demonstrated by splenomegaly, cachexia, lethargy or dermal/ocular abnormalities, were detected. The transgenic human CD4, expressed in addition to the murine orthologue, did not appear to be pivotal for diabetes development (ESM Fig. 1a). All mice with diabetes demonstrated severe immune infiltrate in the pancreatic islets. This infiltrate was highly diverse, with both lymphoid and myeloid cells, expressing CD4, CD8, B220, CD11b and CD11c, found in abundance in all islets (Fig. 1b). Ly6G ${ }^{+}$ granulocytic cells were found in lower numbers in some islets. No substantial immune cell infiltration was observed in the non-diabetic mice that we examined histologically at the age of 6-8 weeks $(n=5), 10$ weeks $(n=10), 12$ weeks $(n=7), 16-$
20 weeks $(n=5)$ or even 35 weeks $(n=13)$, bar one 6-weekold male mouse in which moderate infiltration of some islets was detected (ESM Fig. 1b). This suggests that the insulitic phenotype is strongly associated with disease and perhaps reflects a rapid progression to frank diabetes. Because the onset of type 1 diabetes is characterised by the presence of autoantibodies against GAD65 and insulin in most HLADR3-DQ2 humans, sera of mice were tested for antibodies against murine 30-mer peptides that span the length of murine proinsulin-2, the isoform most similar to human proinsulin, and against human GAD65, which has over 95\% sequence homology with the murine equivalent. As depicted in Fig. $1 \mathrm{c}$, e, there was a significant increase in the levels of antiproinsulin-2, but not anti-GAD65, antibodies when groups of mice at increasing ages were compared. There was a spread of levels of anti-GAD65 antibodies; a small number of mice displayed high binding, particularly at early ages. There did not appear to be any direct link between the appearance of these autoantibodies and the development of diabetes in individual mice. However, it should be noted that insulin autoantibodies detected in human disease typically recognise the 
Fig. 3 Murine proinsulin-2 peptide B23-C20 mediates diabetogenicity. (a)

DR3DQ2 $\times$ RIP-B7.1 mice aged

7-8 weeks ( 3 male, 3 female mice in each group) were primed with $100 \mu \mathrm{g}$ of either murine 30-mer proinsulin-2 peptide B1-30, B23$\mathrm{C} 20, \mathrm{C} 10-\mathrm{A} 6$ or $\mathrm{C} 25-\mathrm{A} 21$ in

TiterMax Gold adjuvant s.c. on days 0 and 14 , with $200 \mathrm{ng}$ pertussis toxin administered i.p on days 0 and 1 or 2 . Mice were monitored weekly for diabetes up to 20 weeks post prime. The grey dashed line indicates the expected level of spontaneous diabetes in mice aged 8 weeks on day 0 of the experiment (based on the data in Fig. 1 and normalised for sex). $* * * p<0.001$ for B23-C20 vs C10-A6; ${ }^{\dagger \dagger} p<0.01$ for B23-C20 vs $\mathrm{C} 25-\mathrm{A} 21 ;{ }^{+1} p<0.001$ for B23-C20 vs B1-30; other comparisons not significant (Mantel-Cox logrank test). (b-e) Blood glucose levels for each individual mouse in the group immunised with B1-30 (b), B23$\mathrm{C} 20$ (c), C10-A6 (d) or C25-A21 (e); key in (b) also applies to (c-e) a
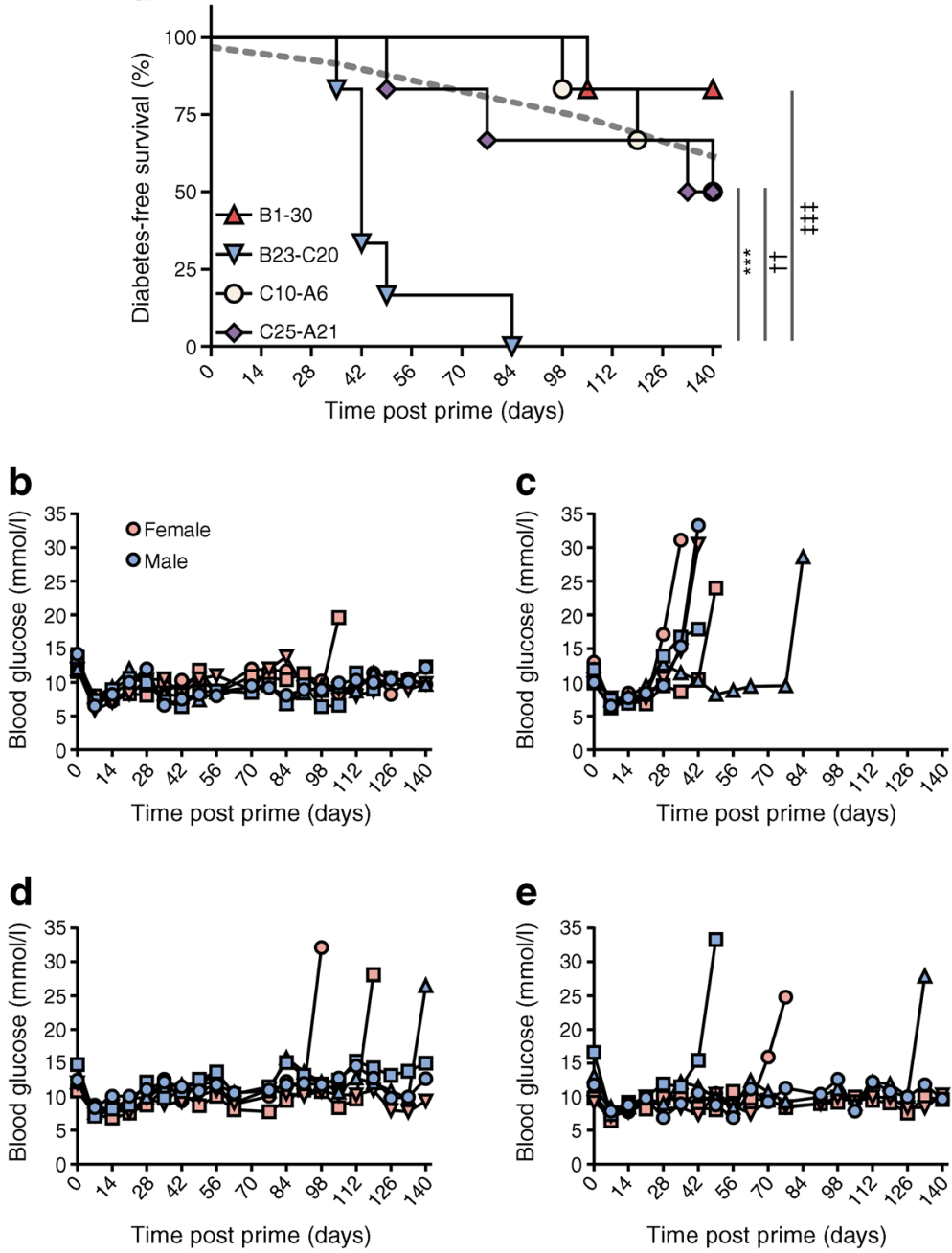

secondary structure of insulin protein. Future work will be required to assess whether the use of conformationally intact insulin for autoantibody assays provides additional insights, such as a relationship between antibody presence/levels and disease development. Diabetic DR3DQ2×RIP-B7.1 mice displayed higher levels of autoantibodies compared with DR4×RIP-B7.1 mice that do not develop diabetes spontaneously but this difference did not reach statistical significance (Fig. 1d, f).

Proinsulin is a diabetogenic antigen in DR3DQ2 $\times$ RIP-B7.1 mice The spontaneous onset of diabetes in mice carrying a high-risk transgenic HLA suggests that an autoimmune process is central to disease development and prompted us to address the question of whether particular autoantigens are disease drivers. We tested the hypothesis that priming mice against candidate molecules using adjuvant would accelerate disease progression if the antigens had 'driver' properties. Accordingly, mice were primed with individual 30-mer peptides that overlap and span the length of murine proinsulin-2, recombinant human GAD65, the 377-amino acid intracellular region of human IA-2 or PBS alone in TiterMax Gold adjuvant (Fig. 2a-e). Importantly, of these conditions, only priming with proinsulin peptides clearly hastened diabetes onset and increased incidence beyond that observed with control stimuli or through spontaneous onset. By the end of the experiment at 20 weeks, every mouse primed with proinsulin peptides in adjuvant (10/10) had developed diabetes (mean \pm SD time of onset $77 \pm 38$.6 days post prime). The pancreatic immune infiltrate did not differ in intensity or diversity when comparing these antigen-challenged mice with mice that developed diabetes spontaneously (not shown). These findings show the importance of immune recognition of proinsulin in the context of HLA-DR3-DQ2 as a diabetes-driving event in this model.

A specific region of proinsulin is responsible for diabetes induction in this model We next examined whether there is a dominant region of proinsulin that interacts in DR3DQ $2 \times$ RIPB7.1 mice to promote diabetes by repeating these adjuvanted priming experiments with each of the four proinsulin-2 peptides individually (Fig. 3a-e). Priming with B23-C20 peptide 
provided the most overt disease exacerbation, with very rapid development of diabetes $(100 \%$ incidence by day 84 post prime; mean \pm SD onset $49 \pm 16.2$ days post prime), characterised by severe leucocyte infiltration in the pancreatic islets (ESM Fig. 1b). In contrast, the disease incidence with the remaining three peptides was not markedly different from that expected spontaneously.

To examine how translational these findings might be and understand potential interactions of driver antigens/epitopes with HLA, we first aligned the relevant regions of murine proinsulin-2 and human proinsulin (Fig. 4a). The B23-C20 regions of human proinsulin and murine proinsulin-2 show high homology, with 23/30 amino acids (77\%) being identical and a further $3 / 30(10 \%)$ similar between species. Next, to further pinpoint the region that has the dominant diseaseinducing effect, we generated six overlapping 14/15-mer peptides that span the length of B23-C20. Sequences with Nterminal glutamine $(\mathrm{Q})$ or glutamic acid $(\mathrm{E})$ residues were avoided, to obviate formation of pyroglutamic acid, yielding peptides with unpredictable properties. One of the sequences, RG-15, was highly insoluble in aqueous solution and was therefore not incorporated in further disease-induction experiments. It has an identical predicted binding core to that of

a

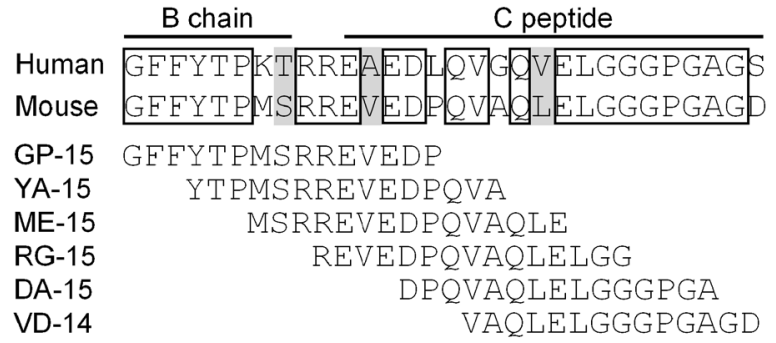

b

\begin{tabular}{|c|c|c|c|c|}
\hline & & \multicolumn{2}{|c|}{ 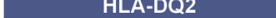 } \\
\hline Peptide & Core & $\mathrm{IC}_{50}(\mathrm{nmol} / \mathrm{I})$ & Core & $\mathrm{C}_{50}(\mathrm{nmol} / \mathrm{I})$ \\
\hline GFFYTPMSRREVEDP & FFYTPMSRR & 490.26 & FFYTPMSRR & 3959.45 \\
\hline YTPMSRREVEDPQVA & REVEDPQVA & 14204.88 & MSRREVEDP & 3737.37 \\
\hline MSRREVEDPQVAQLE & EVEDPQVAQ & 7011.58 & EDPQVAQLE & 1306.68 \\
\hline REVEDPQVAQLELGG & EVEDPQVAQ & 8436.52 & EDPQVAQLE & 1040.49 \\
\hline DPQVAQLELGGGPGA & PQVAQLELG & 19605.27 & PQVAQLELG & 7097.60 \\
\hline (Q)VAQLELGGGPGAGD & LELGGGPGA & 23175.96 & LELGGGPGA & 16095.38 \\
\hline
\end{tabular}

C

\begin{tabular}{|l|c|l|l|l|}
\hline \multicolumn{1}{|c|}{ Peptide } & \multicolumn{2}{c}{ Core } & IC $_{50}(\mathrm{nmol} / \mathrm{I})$ & \multicolumn{2}{c|}{ Core } & IC $_{50}(\mathrm{nmol} / \mathrm{l})$ \\
\hline GFFYTPKTRREAEDL & FFYTPKTRR & 341.18 & KTRREAEDL & 7594.00 \\
\hline YTPKTRREAEDLQVG & TRREAEDLQ & 12139.06 & TRREAEDLQ & 2681.41 \\
\hline KTRREAEDLQVGQVE & EAEDLQVGQ & 10335.91 & EDLQVGQVE & 1069.04 \\
\hline REAEDLQVGQVELGG & LQVGQVELG & 6776.19 & EDLQVGQVE & 1073.75 \\
\hline DLQVGQVELGGGPGA & LQVGQVELG & 10940.62 & LQVGQVELG & 6190.23 \\
\hline (Q)VGQVELGGGPGAGS & VELGGGPGA & 25844.08 & VELGGGPGA & 18725.77 \\
\hline
\end{tabular}

Fig. 4 Analysis and comparison of proinsulin-2 B23-C20. (a) Alignment of murine proinsulin-2 with the equivalent region of human proinsulin. Boxed amino acids are identical, shaded regions are similar. Six overlapping 14- to 15-mer murine peptides generated for more detailed analysis are shown. For VD-14 peptide, the preceding glutamine (Q) in the sequence was added for the prediction, to reach the minimum 15-aminoacid length required. (b) Binding predictions for HLA-DR3 and HLADQ2 of all six murine peptides. (c) Binding predictions for HLA-DR3 and HLA-DQ2 of human equivalents of the murine peptides
ME-15 for both HLA-DR3 and HLA-DQ2 (Fig. 4b). Based on the predicted binding affinity, none of the 14/15-mer peptides were classed as strong binders to HLA-DR3 or HLADQ2 by the prediction tool used. Importantly, the predicted binding core and affinity for the human equivalents of our peptides was generally comparable with mouse sequences (Fig. 4c).

Adjuvanted priming with the ME-15 (B29-C11) peptide led to markedly higher disease incidence during follow-up than would be expected spontaneously. Disease was also significantly accelerated compared with any of the other 14/15mer peptides tested in this experiment (Fig. 5a-f), strongly suggesting that only the ME-15 peptide contains the minimum core amino acid sequence that binds HLA and interacts with immune cells to drive diabetes.

Finally, we conducted a small pilot study using an ELISPOT assay (see ESM Methods) to analyse IFN- $\gamma$ secretion by human peripheral blood mononuclear cells (PBMCs) in response to the human proinsulin peptide $\mathrm{B} 30-\mathrm{C} 13$, which was previously reported to have the same predicted HLA-DQ binding region as B29-C11 [12]. This suggested that HLADR3-DQ2 donors, but not those with other HLA haplotypes, exhibit responses to B30-C13 that were greater than those to the control, HLA-DR4-restricted [13], peptide C19-A3 (ESM Fig. 2a, b). These preliminary data will require confirmation in a larger series.

\section{Discussion}

The novel DR3DQ2 $\times$ RIP-B7.1 model presented here develops diabetes spontaneously, in contrast to the DR4 $\times$ RIPB7.1 model we reported previously, which is only susceptible to induced disease [11]. Although the different approaches to the creation of these two HLA-transgenic models (chimeric vs native), and the fact that the HLA-DQ8 homologue is missing from the latter, makes direct comparison difficult, it seems likely that the inclusion of HLA-DQ2 from the DR3 highrisk haplotype is an important feature in the new model. HLA-DQ2, similar to HLA-DQ8, has previously been found to be linked strongly to diabetes susceptibility [14]. It is encouraging for the future use of inducible models of type 1 diabetes that the immune infiltrate in the pancreatic islets is very similar in the spontaneous-onset DR3DQ2 $\times$ RIP-B7.1 model and the induced DR4 $\times$ RIP-B7.1 model and that the diversity of immune cells infiltrating is reminiscent of human type 1 diabetes and other major preclinical models such as the non-obese diabetic (NOD) mouse [15]. The fact that we struggled to intercept pancreases at a point of moderate insulitis without established diabetes in DR3DQ2 $\times$ RIP-B7.1 mice suggests that disease progression in this model may be very rapid from the point of first immune infiltration to established diabetes. Despite the fact that autoantibodies to proinsulin 
Fig. 5 ME-15 peptide (B29-C11) covers the core region of B23-

C20 for diabetogenicity. (a)

DR3DQ2×RIP-B7.1 mice aged

$10-14$ weeks were primed with

$100 \mu \mathrm{g}$ of either GP-15 (2 male, 3

female mice), YA-15 (2 male, 3

female mice), ME-15 (3 male, 2

female mice), DA-15 (2 male, 3

female mice) or VD-14 (3 male, 2

female mice) peptide in TiterMax

Gold adjuvant s.c. on days 0 and

14 , with $200 \mathrm{ng}$ pertussis toxin

administered i.p on days 0 and 1

or 2. Mice were monitored

weekly for diabetes up to

20 weeks post prime. The grey

dashed line indicates the expected

level of spontaneous diabetes in

mice aged 14 weeks on day 0 of

the experiments (based on the

data in Fig. 1 and normalised for sex). $* * p<0.01$ for ME-15 vs

GP- $15 ;{ }^{\dagger} p<0.05$ for ME-15 vs

DA-15; ${ }^{*} p<0.01$ for ME-15 vs

VD- $14 ;{ }^{\S} p<0.01$ for ME-15 vs

YA-15; other comparisons not significant (Mantel-Cox logrank test). (b-f) Blood glucose levels for each individual mouse in the group immunised with GP-15 (b), YA-15 (c), ME-15 (d), DA-15 (e) or VD-14 (f); key in (b) also applies to $(\mathbf{c}-\mathbf{f})$
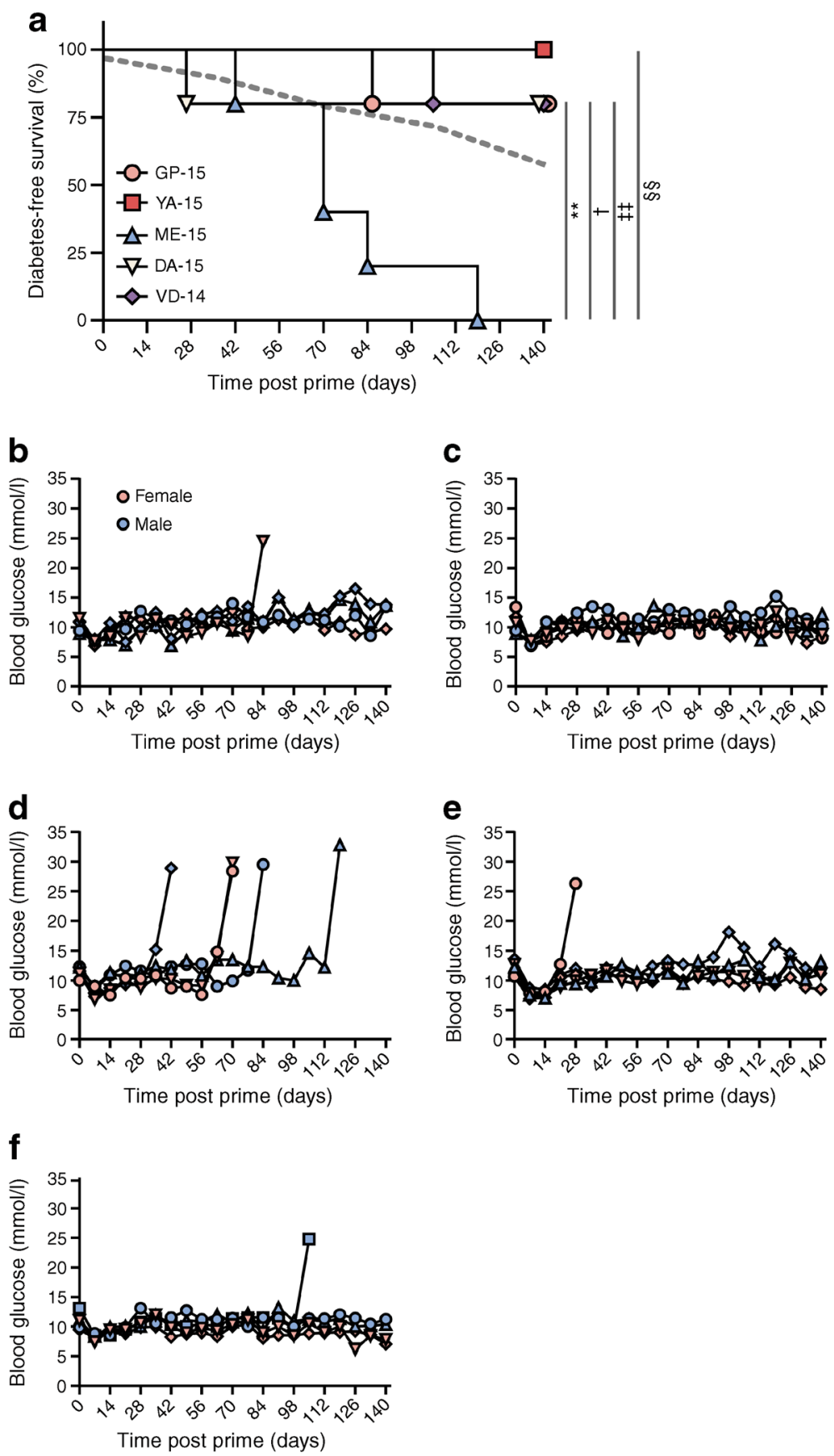

(and in the DR3DQ2 $\times$ RIP-B7.1 model, also GAD65) could be detected, these did not appear to be directly linked to the development of diabetes in either model.

The finding that only priming with the murine proinsulin-2 peptides led to the development of diabetes was somewhat unexpected. Previous studies have predominantly identified GAD65 and IA-2 to be the target islet antigens recognised by $\mathrm{CD}^{+} \mathrm{T}$ cells or $\mathrm{T}$ cell clones from individuals with type 1 diabetes using the HLA-DR3 and -DQ2 restriction elements [4]. Moreover, the TEDDY study suggests that anti-GAD65 autoantibodies are typically the first to appear in diseasesusceptible individuals with the HLA-DR3-DQ2 haplotype.
In contrast, anti-insulin autoantibodies usually emerge first in at-risk individuals with the HLA-DR4-DQ8 haplotype [8, 9]. These represent potentially important disease endotypes, characterised by distinct pathological processes involving loss of immunological tolerance to GAD65 and insulin, respectively. In light of this, it might have been predicted that GAD65 would be the most potent antigenic driver of disease in the new DR3DQ2 $\times$ RIP-B7.1 model, and yet this turned out not to be the case. There are a few caveats to our experiments, however, that should limit overinterpretation, including the fact that the GAD65 and IA-2 antigens we used (the only ones available for experimentation) are human in origin. Although 
highly similar to their murine orthologues, there may be critical differences within key immunogenic regions. Moreover, for IA-2 only the intracellular region, but not the full-length protein, is available as a soluble recombinant reagent. Intracellular IA-2 is known to be an important autoantigen for serological and $\mathrm{T}$ cell responses [13, 16], although the extracellular section also contains regions demonstrated to be immunogenic and could thus play a role in diabetes pathology [17]. Finally, protein (fragment), as used to study the response to GAD65 and IA-2, may be intrinsically less efficient than short linear peptides (e.g. as those used for proinsulin-2) at priming, breaking tolerance and driving disease. It would therefore be inappropriate to conclude categorically that GAD65 and IA-2 are not able to initiate diabetes in our model or that spontaneous disease must be mediated by targeting proinsulin alone. Nonetheless, the results are intriguing and could be consistent with there being a disconnect between the disease importance of antigens that drive early serological responses and those that drive cell-mediated beta cell destruction. Such a hypothesis could be examined in highrisk groups with particular HLA haplotypes and that are followed longitudinally from birth.

A recent study suggested that secretory and crinophagic granules of human beta cells contain only a limited array of proinsulin-derived peptides [18]. C-peptide sequences feature heavily but all of these are from the $\mathrm{N}$-terminal region. $\mathrm{N}$-terminal C-peptide also includes the predicted core HLA-DR3- and HLA-DQ2-binding regions of our diabetogenic ME-15 (B29-C11) peptide. It is, therefore, tantalising to speculate that the dominance in the generation of peptides from this proinsulin region by beta cells themselves may be responsible for their demise in HLA-DR3DQ2-bearing people with type 1 diabetes. To our knowledge, peptides from the B23-C20 region of proinsulin, murine or human, have not previously been identified as being either HLA-DR3- or HLA-DQ2-restricted. Furthermore, no previous study has directly demonstrated diabetogenicity of this region in association with any haplotype; indeed, the approach of using antigen-adjuvanted priming against selected HLA backgrounds to explore the potential diabetogenicity and relative primacy of antigens and epitopes is novel as far as we are aware. For HLA$\mathrm{DQ}^{+}{ }^{+}$individuals, or the structurally highly similar I-A ${ }^{\mathrm{g} 7}$ MHC molecule in NOD mice, the B9-23 region of proinsulin is potentially important for diabetes induction [19]. In contrast, in our DR4-RIP-B7.1 model we demonstrated that disease could only be induced with proinsulin $\mathrm{C} 25$ A21 peptide [11], which represents a region known to be immunogenic in human type 1 diabetes [13]. Thus, where appropriate studies have been performed, a consensus on the HLA-specific immunogenic regions of proinsulin is emerging and it remains highly plausible that the $\mathrm{B}-\mathrm{C}$ junction is important for HLA-DR3/DQ2-associated disease. Few studies have examined T cell reactivity to this region, and fewer still for HLA-DR3/DQ2 restricted responses. Semana et al previously found HLA-DRrestricted $\mathrm{CD}^{+} \mathrm{T}$ cell responses to proinsulin peptide C3-16, which overlaps significantly with the region we identify here, in individuals with diabetes but these were not analysed for HLA [20]. In autoantibody-positive individuals of the $H L A-D R B 1 * 04 D Q B 1 * 0302$ haplotype, the long B11-C24 peptide is recognised frequently [21]. So et al identified $\mathrm{CD}^{+} \mathrm{T}$ cell responses to $\mathrm{C} 2-11$ and $\mathrm{C} 3$ 14 in three out of their $22 \mathrm{~T}$ cell clones, albeit with HLADQ8 restriction [7]. HLA-DQ2-restricted peptides able to generate a $\mathrm{T}$ cell response were all located near the $\mathrm{C}$ terminal end of C-peptide. As discussed by the authors of that study, the limitation of such studies is that $\mathrm{T}$ cell reactivity does not necessarily imply diabetogenicity in vivo.

The new model provides opportunities to gain deeper insight into the pathology and enhance translatability. First, it will be important to identify and characterise antigen-specific $\mathrm{CD}^{+} \mathrm{T}$ cells in both spontaneous and accelerated disease. Second, additional modifications to the model could be introduced to potentially augment humanisation (e.g. by transgenic replacement of murine proinsulin with its human equivalent, allowing the direct use of human proinsulin sequences to drive the pathological processes).

In summary, we here provide in vivo evidence for the identification of a disease-relevant region within proinsulin that could have ramifications for understanding disease development in individuals with type 1 diabetes and bearing the most common HLA haplotype, HLA-DR3-DQ2.

Data availability All relevant data are available in this article and ESM. Reasonable requests for access to the in vivo models described can be made to the corresponding author.

Funding This work was funded by UCB Pharma Ltd. The sponsor was involved in study design and decision to submit for publication only. Related work in our laboratory receives funding from the Innovative Medicines Initiative 2 Joint Undertaking under grant agreement no. 115797 INNODIA. This Joint Undertaking receives support from the European Union's Horizon 2020 research and innovation programme and 'EFPIA', 'JDRF International' and 'The Leona M. and Harry B. Helmsley Charitable Trust'.

Duality of interest ES is an employee of UCB Pharma Ltd. who were developing peptide immunotherapy for type 1 diabetes between 2014 and 2018 through a licence agreement with King's College London. MP is employed by King's College London and during the period specified received research funds and provided consultancy services for UCB. All other authors declare that there is no duality of interest associated with their contribution to this manuscript.

Contribution statement JV designed and conducted experiments, analysed data and wrote the manuscript. EMW and KN conducted in vivo experiments and analysed relevant data. NY and SA designed and conducted the human experiment and analysed relevant data. ELS 
and MP provided feedback and guidance regarding the design of the study. All authors revised the manuscript critically for important intellectual content and approved the final version of the manuscript. JV is responsible for the integrity of the work as a whole.

Open Access This article is distributed under the terms of the Creative Commons Attribution 4.0 International License (http:// creativecommons.org/licenses/by/4.0/), which permits unrestricted use, distribution, and reproduction in any medium, provided you give appropriate credit to the original author(s) and the source, provide a link to the Creative Commons license, and indicate if changes were made.

\section{References}

1. Huo L, Harding JL, Peeters A, Shaw JE, Magliano DJ (2016) Life expectancy of type 1 diabetic patients during 1997-2010: a national Australian registry-based cohort study. Diabetologia 59(6):11771185. https://doi.org/10.1007/s00125-015-3857-4

2. Petrie D, Lung TW, Rawshani A et al (2016) Recent trends in life expectancy for people with type 1 diabetes in Sweden. Diabetologia 59(6):1167-1176. https://doi.org/10.1007/s00125-016-3914-7

3. Erlich H, Valdes AM, Noble J et al (2008) HLA DR-DQ haplotypes and genotypes and type 1 diabetes risk: analysis of the type 1 diabetes genetics consortium families. Diabetes 57(4):1084-1092. https://doi.org/10.2337/db07-1331

4. Di Lorenzo TP, Peakman M, Roep BO (2007) Translational minireview series on type 1 diabetes: systematic analysis of $\mathrm{T}$ cell epitopes in autoimmune diabetes. Clin Exp Immunol 148(1):1-16. https://doi.org/10.1111/j.1365-2249.2006.03244.x

5. Narendran P, Mannering SI, Harrison LC (2003) Proinsulin-a pathogenic autoantigen in type 1 diabetes. Autoimmun Rev 2(4):204 210. https://doi.org/10.1016/S1568-9972(03)00009-0

6. Zhang L, Nakayama M, Eisenbarth GS (2008) Insulin as an autoantigen in NOD/human diabetes. Curr Opin Immunol 20(1): 111-118. https://doi.org/10.1016/j.coi.2007.11.005

7. So M, Elso CM, Tresoldi E et al (2018) Proinsulin C-peptide is an autoantigen in people with type 1 diabetes. Proc Natl Acad Sci U S A 115(42):10732-10737. https://doi.org/10.1073/pnas. 1809208115

8. Krischer JP, Lynch KF, Lernmark A et al (2017) Genetic and environmental interactions modify the risk of diabetes-related autoimmunity by 6 years of age: the TEDDY study. Diabetes Care 40(9): 1194-1202. https://doi.org/10.2337/dc17-0238

9. Krischer JP, Lynch KF, Schatz DA et al (2015) The 6 year incidence of diabetes-associated autoantibodies in genetically at-risk children: the TEDDY study. Diabetologia 58(5):980-987. https://doi.org/10. 1007/s00125-015-3514-y

10. de Kauwe AL, Chen Z, Anderson RP et al (2009) Resistance to celiac disease in humanized HLA-DR3-DQ2-transgenic mice expressing specific anti-gliadin $\mathrm{CD}^{+} \mathrm{T}$ cells. J Immunol 182(12): 7440-7450. https://doi.org/10.4049/jimmunol.0900233

11. Verhagen J, Smith EL, Whettlock EM, Macintyre B, Peakman M (2018) Proinsulin-mediated induction of type 1 diabetes in HLADR4-transgenic mice. Sci Rep 8(1):14106. https://doi.org/10.1038/ s41598-018-32546-4

12. van Lummel M, van Veelen PA, de Ru AH et al (2016) Discovery of a selective islet peptidome presented by the highest-risk HLADQ8trans molecule. Diabetes 65(3):732-741. https://doi.org/10. 2337/db15-1031

13. Arif S, Tree TI, Astill TP et al (2004) Autoreactive T cell responses show proinflammatory polarization in diabetes but a regulatory phenotype in health. J Clin Invest 113(3):451-463. https://doi.org/ 10.1172/JCI19585

14. Noble JA (2015) Immunogenetics of type 1 diabetes: a comprehensive review. J Autoimmun 64:101-112. https://doi.org/10.1016/j. jaut.2015.07.014

15. Willcox A, Richardson SJ, Bone AJ, Foulis AK, Morgan NG (2009) Analysis of islet inflammation in human type 1 diabetes. Clin Exp Immunol 155(2):173-181. https://doi.org/10.1111/j. 1365-2249.2008.03860.x

16. Peakman M, Stevens EJ, Lohmann T et al (1999) Naturally processed and presented epitopes of the islet cell autoantigen IA-2 eluted from HLA-DR4. J Clin Invest 104(10):1449-1457. https://doi. org/10.1172/JCI7936

17. van Lummel M, van Veelen PA, de Ru AH et al (2016) Dendritic cells guide islet autoimmunity through a restricted and uniquely processed peptidome presented by high-risk HLA-DR. J Immunol 196(8):3253-3263. https://doi.org/10.4049/jimmunol.1501282

18. Wan X, Zinselmeyer BH, Zakharov PN et al (2018) Pancreatic islets communicate with lymphoid tissues via exocytosis of insulin peptides. Nature 560(7716):107-111. https://doi.org/10.1038/s41586018-0341-6

19. Daniel D, Gill RG, Schloot N, Wegmann D (1995) Epitope specificity, cytokine production profile and diabetogenic activity of insulin-specific $\mathrm{T}$ cell clones isolated from NOD mice. Eur $\mathrm{J}$ Immunol 25(4):1056-1062. https://doi.org/10.1002/eji. 1830250430

20. Semana G, Gausling R, Jackson RA, Hafler DA (1999) T cell autoreactivity to proinsulin epitopes in diabetic patients and healthy subjects. J Autoimmun 12(4):259-267. https://doi.org/10.1006/ jaut.1999.0282

21. Durinovic-Bello I, Boehm BO, Ziegler AG (2002) Predominantly recognized proinsulin $T$ helper cell epitopes in individuals with and without islet cell autoimmunity. J Autoimmun 18(1):55-66. https:// doi.org/10.1006/jaut.2001.0566

Publisher's note Springer Nature remains neutral with regard to jurisdictional claims in published maps and institutional affiliations. 